\title{
El proceso de educación ambiental y su expresión en el contexto rural
}

\author{
Juan Carlos Rodríguez Cruz \\ Universidad Oscar Lucero, Ciudad, Holguín (Cuba)
}

Luiz Bezerra Neto

Universidade Federal de São Carlos

\section{Resumen}

El trabajo aborda la problemática ambiental desde diferentes referentes. Se analiza la categoría medio ambiente y su connotación a partir de la imbricación con la educación, medio idóneo que permite una transformación de un estado real a uno deseado. Se conceptualiza la educación ambiental a partir de su contenido definitorio desde una vertiente pedagógica, con expresiones prácticas en contextos rurales cubanos que permite el rescate de tradiciones y costumbres campesinas.

Palabras claves: Educación. Contexto Rural. Campesino. Medio Ambiente. 


\section{The process of environmental education and its expression in the rural context}

This work analyzes the environmental problems from different sources related to them. The category environment and its connotation are analyzed through their close relation to education, an appropriate means that enables a transformation from a real state to desired one. The environmental education is conceptualized starting from its defining content from a pedagogic source, with practical expressions in rural contexts cubans that enable the rescue of rural customs and traditions.

Keywords: Education. Context Rural. Peasant. Environment.

\section{O processo de educação ambiental e a sua expressão no contexto rural}

O presente trabalho engloba a problemática ambiental, partindo de diferentes abordagens. Analisa a categoria meio ambiente e a sua conotação interligando-a à educação, meio idôneo, que permite uma transformação de um estado real para um almejado. Conceitualiza a educação ambiental a partir de seu conteúdo de definição, partindo de uma vertente pedagógica, com expressões prática sem contextos rurais cubanos que permitem o resgate de tradições e costumes camponeses.

Palavras chave: Educação. Contexto Rural. Camponês. Meio Ambiente. 


\section{Introducción}

El mundo contemporáneo vive hoy una crisis ecológica de la que no escapa Cuba, la misma se expresa en el plano agrícola, con disminución constante de espacios cultivables y especies, a causa de la elevación del nivel del mar, desertificación, salinización, contaminación y erosión de los suelos, polución de las aguas, entre otras. Esta crisis, es consecuencia de un inadecuado tratamiento de la tierra y las aguas superficiales y subterráneas, que incluye el uso indiscriminado, sin referencias técnicas, de fertilizantes y pesticidas, a la vez que se abandonan el uso de técnicas tradicionales de producción con fuerte contenido ambiental.

La problemática se hace compleja por la ausencia de una educación ambiental contextualizada con los problemas ambientales que presentan las distintas comunidades rurales, capaz de incidir directamente sobre la población campesina productora, cuya mayoría no participa de la educación formal. En este medio, la educación no formal juega un papel protagónico, pues es capaz de aportar las herramientas pedagógicas necesarias para la preparación de grupos sociales específicos, como es el caso de una población campesina necesitada de una educación ambiental.

A lo largo del último siglo y en un proceso que se ha acelerado después del triunfo de la Revolución Cubana, a partir de 1959, la población cubana se ha transformado en una población predominantemente urbana, no obstante, se conserva una comunidad campesina portadora de una identidad peculiar dentro del contexto cultural; sin embargo, esta identidad no ha sido suficientemente estudiada, en especial, respecto de las formas tradicionales de producción.

Por otro lado, es inadmisible negarse a reconocer que las modificaciones introducidas en los campos cubanos, con la creación de nuevas formas de producción, han tenido un impacto en la cultura del trabajo agrícola tradicional. Esto ha traído consigo, en algunos casos, aportes positivos, mientras que en otros han generado una ruptura de las tradiciones culturales, que juegan un papel importante en la conservación del medio ambiente rural y de la identidad campesina.

En los contextos rurales holguineros de Gibara y San Andrés, Cuba, se está produciendo un deterioro paulatino del medio ambiente debido, fundamentalmente, a la aplicación incontrolada de fertilizantes y pesticidas, a lo que se agregan los residuales, en particular, de la industria de materiales de la construcción que no están tomando las medidas suficientes para reducir la agresión al medio ambiente.

A partir de lo anteriormente planteado se traza como objetivo del trabajo realizar una valoración del proceso de educación ambiental con expresión en los contextos rurales antes mencionados, tomando como punto de partida diferentes conceptualizaciones sobre medio ambiente, así como el tratamiento a la categoría educación no formal como vía para la praxis social pedagógica. 


\section{Desarrollo}

Es imposible valorar la educación ambiental sin tratar el concepto medio ambiente. Este término era un vocablo asociado al medio natural hasta inicios de la década de los 70 del siglo $X X$ y ha ido evolucionando al incorporársele aspectos sociales. Al respecto Novo (1998), señala que el medio natural comienza a ser denominado medio ambiente en un proceso de enriquecimiento semántico muy clarificador.

En la Conferencia Intergubernamental de educación ambiental, celebrada en Tbilisi (1977), se incluyeron en el concepto elementos de naturaleza socioculturales. En los documentos de la Conferencia se señala que el medio ambiente no solo debe abordar el medio físico, sino que debe tomar en consideración las interacciones entre el medio natural, sus componentes biológicos y sociales con los factores culturales. En correspondencia con lo anterior Novo (1998), concibe al medio ambiente como un sistema complejo y dinámico de interacciones ecológicas, socioeconómicas y culturales que evolucionan, a través del proceso histórico de la humanidad.

La Ley 81 del Medio Ambiente de la República de Cuba (2004, p.3), lo define como: "el sistema de elementos abióticos, bióticos y socioeconómicos, con el que interactúa el hombre, a la vez que se adapta al mismo, lo transforma y lo utiliza para satisfacer sus necesidades."

En esta definición no se explicita el componente cultural del medio ambiente, no obstante la Estrategia Nacional de Medio Ambiente (2007, p. 6), presenta una definición más abarcadora, al reconocer a este como: "un sistema complejo y dinámico de interacciones ecológicas, socioeconómicas y culturales que evoluciona, a través del proceso histórico de la sociedad". El autor de la investigación se acoge a este enunciado.

El problema ambiental es inherente a la sociedad y solo el cambio de actitudes realizado conscientemente por los sujetos sociales, puede producir una modificación significativa de los comportamientos que lo provocan y esto solo es posible a través de la educación ambiental.

El término de educación ambiental aparece en los documentos de la Organización de las Naciones Unidas para la Ciencia, la Educación y la Cultura (Unesco), a partir de 1965, pero no es hasta 1972, durante la Conferencia de las Naciones Unidas sobre el Medio Humano, celebrada en Estocolmo, donde es definida la educación ambiental como uno de los factores más importantes para establecer relaciones sostenibles en el planeta. En este momento se le da la tarea a la Unesco de establecer un programa internacional de educación ambiental.

En esta conferencia se reconoció que tanto el medio natural como el modificado son igualmente esenciales para el bienestar de la humanidad, de esta forma aparece una comprensión de lo ambiental que rebasa los límites de lo estrictamente natural 
e incorpora la preocupación por el patrimonio histórico cultural. En consonancia con lo anterior, en 1973, se constituyó el programa de las Naciones Unidas para la Conservación del Medio Ambiente (Pnuma), con el consiguiente impulso a la educación ambiental. En 1975 se crea el Programa Internacional de Educación ambiental (Piea).

En 1977 la Unesco llamó, en colaboración con el Pnuma, a la convocatoria de la Primera Conferencia Intergubernamental de educación ambiental que se desarrolló en Tbilisi, Georgia, la cual es considerada como uno de los acontecimientos más significativos en la historia de la educación ambiental. En ella se aprobó, por consenso, una declaración que contenía elementos muy avanzados de educación ambiental:

La educación ambiental debe impartirse a personas de todas las edades y niveles educacionales, en los marcos de la educación formal, no formal e informal, con carácter permanente, acorde con los cambios que se producen en un mundo en rápida evolución.

La educación ambiental debe estar orientada a preparar a los individuos para que enfrenten los problemas sobre una base de solidaridad, sustentados en la ética y en los planos local, nacional e internacional.

En el año 1983 se constituyó la Comisión Mundial del Medio Ambiente y Desarrollo, dirigida al estudio sistémico de los problemas ambientales que afectan al planeta en su conjunto y a finales de la década de los ochenta se vincula la educación ambiental con el desarrollo sustentable. Este último hecho ha sido tan significativo, que en la actualidad difícilmente se concibe la educación ambiental sin esta connotación.

En junio de 1992 se celebra la Cumbre de la Tierra o Cumbre de Río de Janeiro (Conferencia de las Naciones Unidas Sobre Medio Ambiente y Desarrollo), que comprendió un encuentro de alto nivel, a la que asistieron representantes de 178 países, entre ellos más de cien jefes de estado y de gobierno. En la cumbre se aprobaron documentos como: el Tratado de Biodiversidad, el Documento sobre Cambio Climático, la Declaración de los Bosques y la Declaración de Río sobre Medio Ambiente y Desarrollo y se dedicó un capítulo a la educación ambiental en relación con el desarrollo sostenible.

En 1997 se reunieron en Kyoto, Japón, representantes de los países integrantes de la Convención Marco de las Naciones Unidas sobre el Cambio Climático, creada en el seno de la Cumbre de la Tierra, celebrada en Río de Janeiro en 1992 y se elaboró un documento denominado Protocolo de Kyoto. El mismo establecía que los países desarrollados debían reducir sus emisiones de gases causantes del efecto invernadero en un 5,2\% para el año 2012, respecto de las emisiones del año 1990. Este protocolo no fue firmado por el país que emite más gases de efecto invernadero: Estados Unidos. 
Entre el 26 de agosto y el 3 de septiembre de 2002 se efectuó la Conferencia Mundial sobre Desarrollo Sostenible en Johannesburgo, Sudáfrica, que adoptó la forma de Cumbre Mundial sobre este tema. Entre las actividades desarrolladas sobresalen: la evaluación del impacto de la globalización en la degradación ambiental y las diferencias económicas entre el Norte y el Sur así como la desigualdad social como factores que inciden en un desarrollo global sostenible.

Eventos posteriores dirigidos a actualizar este protocolo se efectuaron en Copenhague (2009) Cancún (2010) y Durban (2011). Estos terminaron en un rotundo fracaso por falta de acuerdos entre los países más industrializados.

En el año 2009 se logró que la Asamblea General de la Organización de Naciones Unidas aprobara una resolución denominada Armonía con la Naturaleza, presentada por Bolivia con el apoyo de 62 países, que declara el 22 de abril como el Día Internacional de La Madre Tierra. Con posterioridad la Conferencia Mundial de los Pueblos sobre el Cambio Climático y los Derechos de la Madre Tierra celebrada del 20 al 22 de abril del 2010 en Cochabamba, Bolivia, fue una contundente respuesta a la necesidad de garantizar la sostenibilidad ambiental.

A pesar de los esfuerzos realizados no se ha logrado enfrentar con éxito la problemática ambiental. Por ejemplo, en la Segunda Cumbre de Río de Janeiro + 20 (2012), donde se replantearon los principales problemas ambientales que dañan el planeta, no se alcanzaron resultados significativos, producto de la oposición de los países más desarrollados a firmar acuerdos que consideraban lesivos a sus intereses.

En el ámbito nacional, el Estado Cubano ha prestado atención a la conservación del medio ambiente y por consiguiente a la educación ambiental y aunque las medidas tomadas se inician desde el triunfo revolucionario, toman mayor magnitud a partir de la década del noventa del siglo XX. En este contexto la Reforma Constitucional de 2003, modificó el artículo 27 del texto constitucional de 1976, acorde con las nuevas concepciones de medio ambiente. En este artículo reformado queda expresado que:

El Estado protege el Medio Ambiente y los recursos naturales del país y reconoce su estrecha vinculación con el desarrollo económico y social sostenible para hacer más racional la vida humana y asegurar la supervivencia, el bienestar y la seguridad de las generaciones actuales y futuras. Corresponde a los órganos competentes aplicar esta política. Es deber de los ciudadanos contribuir a la protección del agua, la atmósfera, la conservación del suelo, la flora, la fauna y todo el rico potencial de la naturaleza. (2003, p. 18)

La conservación del medio ambiente en Cuba recibió un fuerte impulso a partir de la creación del Ministerio de Ciencia Tecnología y Medio Ambiente (Citma) en 1994. En 1995 comienzan los primeros trabajos de diseño de la Estrategia Ambiental Nacional (EAN), que se prolongó hasta mediados del año 1997, en que resulta 
aprobada. Esta establece los marcos estratégicos ambientales para el desarrollo de estrategias sectoriales. La Estrategia Ambiental Nacional (EAN), continúa siendo el principal instrumento orientador de la política ambiental nacional.

El 11 de julio de 1997 se promulga la Ley 81 (Ley del Medio Ambiente), que tiene como objetivo: establecer los principios que rigen la política ambiental y señalar las normas básicas para regular la gestión ambiental del Estado y las acciones de los ciudadanos y la sociedad en general.

La Ley de Medio Ambiente dedica el capítulo VII a la educación ambiental. Los elementos centrales de este Capítulo pueden definirse del modo siguiente:

- El Citma, en coordinación con el Ministerio de Educación (Mined), el Ministerio de Educación Superior (MES) y otros órganos y organismos, elaborará estrategias de educación ambiental y contribuirá a su implementación, promoviendo la ejecución de programas en todos los sectores de la economía y los servicios, grupos sociales y la población en general.

- El Ministerio de Educación y el Ministerio de Educación Superior, en coordinación con los demás órganos y organismos competentes, perfeccionarán continuamente la introducción de la temática ambiental en el Sistema Nacional de Educación (SNE).

- El Ministerio de Educación Superior garantizará la introducción de la dimensión ambiental, a partir de los modelos del profesional y de los planes de estudio de pregrado, postgrado y de extensión universitaria, actividades docentes y extradocentes, dirigidas a la formación y el perfeccionamiento de los profesionales de todas las ramas.

- Los medios de difusión masiva tendrán la responsabilidad de incorporar en el diseño y la ejecución de su programación televisiva, radial y en prensa plana, los temas ambientales.

- El Ministerio de Ciencia, Tecnología y Medio Ambiente, en coordinación con los Órganos Locales del Poder Popular correspondiente, promoverá y apoyará las actividades educativas en la población, lo que incluye la ejecución de tareas de capacitación y autogestión ambiental comunitaria, vinculadas a las condiciones y necesidades de cada localidad.

El capítulo VIII del Título I de la Ley de Medio Ambiente se dedica a la investigación científica y a la innovación tecnológica y en su artículo 57 dispone: 
Promover los estudios encaminados a ampliar los conocimientos sobre el estado de los recursos naturales y el medio ambiente en general; fomentar y promover la investigación científica y la innovación tecnológica que permitan el conocimiento y desarrollo de nuevos sistemas, métodos, equipos, procesos, tecnologías y dispositivos para la protección del medio ambiente, así como la adecuada evaluación del proceso de transferencia tecnológica.

Desarrollar y aplicar las ciencias y las tecnologías que permitan prevenir, evaluar, controlar y revertir el deterioro ambiental, aportando alternativas de solución a los problemas vinculados a la protección del medio ambiente y promover el uso de tecnologías ambientales adecuadas que armonicen los métodos tradicionales con los requerimientos y exigencias del desarrollo sostenible.

Como se puede apreciar estos aspectos están en plena concordancia con la presente investigación que, a su vez, lleva a la práctica los preceptos de la Ley de Medio Ambiente en un contexto rural.

La Ley de Medio Ambiente tiene un criterio muy estricto de la protección del patrimonio cultural, al asociarlo al entorno natural. De manera especial resalta aquellas circunstancias que puedan poner en peligro el patrimonio cultural; los artículos 144, 145 y 146, otorgan especial atención a la protección del patrimonio cultural. A la vez esta ley plantea en su artículo 18 que,

La política ambiental cubana se ejecuta mediante una adecuada gestión que utiliza diferentes instrumentos entre los que se encuentran: la Estrategia Ambiental Nacional; el Programa Nacional de Medio Ambiente y Desarrollo y los demás programas, planes y proyectos de desarrollo económico y social. La educación ambiental; la investigación científica y la innovación tecnológica.

La educación ambiental, preconizada por la Ley 81, presenta un nuevo enfoque de las relaciones entre el hombre y la manera en que este debe influir en su medio ambiente, con el objetivo primordial de formar ciudadanos conscientes y responsables, con adecuados valores ecológicos y de convivencia. La situación obliga a contextualizar dicha educación a los diferentes sectores y grupos sociales, sobrepasando el marco de la educación formal y aprovechando las amplias posibilidades de la educación no formal. En este contexto se inscribe el presente trabajo dirigido a un grupo social con gran responsabilidad en la degradación de los suelos y la contaminación ambiental: los campesinos productores que practican una agricultura intensiva sin criterio de sostenibilidad.

Acorde con (Díaz, 1999), la educación ambiental es enlace entre la educación en su definición más amplia, que incluye la protección del medio ambiente como elemento esencial del desarrollo y la educación integral con todos sus componentes, orientados hacia problemáticas cuya verdadera magnitud se ha comenzado a comprender recientemente. 
A su vez, McPherson (2002), destaca que la aspiración de la educación ambiental es lograr la formación de la población, de manera que esta, de forma consciente, pueda buscar soluciones a los problemas actuales y trabajar para prevenir los que puedan aparecer en el futuro.

La educación ambiental debe ser comprendida como un proceso educativo permanente, en aras de lograr que los individuos y la sociedad en general, tomen conciencia de su medio y adquieran conocimientos, habilidades y valores, que les permitan desarrollar un papel positivo, tanto individual como colectivo hacia la protección del medio ambiente y el mejoramiento de la calidad de vida humana. La educación ambiental no debe reconocer barreras de edad ni de sistemas educativos, al estar dirigida a reorientar de forma positiva impresiones y valores de respeto al medio ambiente.

La misma tiene como objetivo desarrollar, a través de los sistemas educativos, conductas correctas hacia el entorno. Según Muñoz (2005), citado por Fernández (2008), no basta con educar para la naturaleza, utilizándola como recurso educativo, hay que educar para el medio ambiente. La educación ambiental debe estar orientada de manera, que cada hecho, fenómeno o proceso educativo que se analice y cada acción de intervención que se programe o realice, debe ser considerada como un aspecto de la totalidad de la que forman parte. Solo dentro del sistema que lo incluye, se entiende la significación última de algún aspecto o esfera de la realidad y de las intervenciones socioeducativas sobre esa misma realidad.

A la educación ambiental se le debe concebir como una concepción pedagógica, teórico-práctica, basada en la relación naturaleza-naturaleza, hombre-naturaleza, hombre-hombre y hombre-sociedad, que trascienda cualquier sistema educativo y aborde la dimensión medioambiental, no solo desde el perfil de una disciplina, sino integrado el conocimiento de las ciencias, a partir de la concepción de la relación medio ambiente y desarrollo, donde al individuo se le prepara para convertirse en sujeto consciente y activo del medio ambiente.

En la actualidad existen diversas posiciones teóricas acerca del concepto educación ambiental, esto hace muy difícil su valoración. A continuación se analizan importantes definiciones del mismo. El Acuerdo sobre Educación del Fórum Internacional de Organizaciones no Gubernamentales y Movimientos Sociales (1994, p.30), define a la educación ambiental como:

Un proceso de aprendizaje permanente para sociedades sustentables y responsabilidad global basado en el respeto a todas las formas de vida, que afirma valores y acciones que contribuyen con la transformación humana y social y con la preservación ecológica, estilarte de la formación de sociedades socialmente justas y ecológicamente equilibradas, que conserven entre sí una relación de interdependencia y diversidad, lo que refiere responsabilidad individual y colectiva a nivel local, nacional e internacional.

En la Estrategia Nacional de Educación Ambiental (1997, p.7) se conceptúa que: 
La educación para el desarrollo sostenible (...), que se considera un modelo teórico metodológico y práctico que transciende el sistema educativo tradicional y alcanza la concepción de medio ambiente y desarrollo (...) se le concibe como una educación para el desarrollo sostenible, que se expresa y se planifica a través de la introducción de la dimensión ambiental en los procesos educativos.

Las consideraciones anteriores permiten concebir la educación ambiental como un proceso continuo y permanente, que constituye una dimensión de la educación integral de todos los ciudadanos. Lo anterior se orienta en el proceso de adquisición de conocimientos, desarrollo de hábitos, habilidades, actitudes y formación de valores donde se armonicen las relaciones entre los hombres y entre estos con la naturaleza.

Al respecto, Bedoy (2004, p. 2), plantea que la educación ambiental es: "...una nueva educación que requiere del replanteo de los procesos educativos en su conjunto y desarrollarse en un marco de nuevos enfoques, métodos, conocimientos y nuevas relaciones entre distintos agentes educativos".

El mismo autor destaca que dicha educación comprende:

El medio social y natural como un todo interrelacionado entre sí, vinculando los modelos de crecimiento con un desarrollo integral sustentado en un entorno saludable.

La posibilidad de la penetración en la esencia de los procesos, superando sus apariencias, para permitir un acercamiento crítico integral a la realidad.

Un proceso socioeducativo que favorece la formación de herramientas intelectuales y emotivas conducentes a la acción consciente.

Los métodos apropiados que superen el sueño letárgico en que está sumergido el hombre, para que pueda ser el autor y principal actor de su propia historia.

El español Palma (2005, p.3), señala que la educación ambiental es:

Un proceso de formación y concienciación dirigido a todos los niveles y estratos sociales sobre los problemas del medio ambiente y el desarrollo y sus perspectivas de solución, en su actual conceptualización social del desarrollo sostenible en relación con los problemas ambientales, la población y la calidad de vida. 
J. Seferche, citado por Palma (2005, p.3), expone que la educación ambiental, es:

Un concepto amplio, es concienciación social de los problemas ambientales y en esta labor deben intervenir tanto las escuelas como las familias, la administración pública, los decisores ambientales y, en general, todo el entorno social. La educación ambiental implica la responsabilidad personal del hombre y su participación colectiva.

Una conceptualización abarcadora presentan las mexicanas Aguilera; Piñón, (2005), citado por Fernández (2008), las cuales señalan que la educación ambiental es un proceso formativo cuya finalidad es que las personas sean capaces de construir y aplicar nuevos conocimientos, sustentados en percepciones, actitudes, valores y reflexiones, respecto de la forma en que los seres humanos se relacionan con el medio ambiente. En este contexto la participación comunitaria es fundamental para tratar de mitigar y prevenir algunos de los problemas ambientales vigentes; aunque la educación ambiental, no puede resolver los problemas ambientales si no cuenta con el apoyo de decisiones de orden social, económico y político.

La valoración de las definiciones anteriores permite destacar elementos comunes, entre los que resaltan la educación ambiental como un proceso formativo, permanente e integral que trasciende el sistema educativo tradicional y se extiende a toda la sociedad, situación unida a la necesidad de un nuevo enfoque de la misma, que incluya el replanteamiento de los procesos educativos en su conjunto.

A continuación, sustentado en Fernández (2008), se plantean diversas perspectivas sobre la educación ambiental:

Enfoque ético: se reconoce como un proceso de formación de valores para fomentar actitudes y aptitudes dirigidas a la protección del medio ambiente. La educación ambiental establece un nuevo sistema de valores que contempla la condición física del entorno relacionada con el contexto económico social y político.

Enfoque sistémico: el cual se contempla como un proceso sustentado en las interrelaciones entre el medio ambiente social y el natural.

Enfoque metodológico: concebido como una nueva modalidad educativa, en la que la dimensión ambiental se comporta como eje transversal.

Enfoque participativo: que permite a las personas tomar conciencia de los problemas que las afectan de forma individual y colectiva, aclarar las causas de los mismos y buscar los medios para su solución, en un proceso en que participan los distintos elementos de la población en la transformación de su realidad.

Enfoque crítico: propicia la valoración constante de la realidad biofísica y social, estimulando la creatividad en la propuesta de soluciones a la problemática existente, 
estas propuestas deben ser sometidas a un permanente análisis crítico, de manera que resulte un acercamiento integral a la realidad en su constante transformación.

Enfoque holístico: en calidad de proceso integral social, político y pedagógico, en el que deben intervenir los sistemas educativos, la familia y la administración pública, los decisores ambientales y en general todo el entorno social.

Enfoque comunitario: dirigido a la resolución de problemas ambientales concretos de una comunidad, sustentada en el conocimiento y las herramientas que favorezcan la solución en la práctica de estos problemas. Este enfoque, que debe tener presente la cultura y las tradiciones de la comunidad, supera las etapas escolares o académicas para alcanzar todo momento o circunstancia del acontecer cotidiano.

Enfoque cognitivo: donde la adquisición de conocimientos se produce y reproduce en un proceso ascendente de concienciación social de los problemas ambientales. Se trata de utilizar métodos apropiados, que despierten en las personas la necesidad de ser autores y principales actores de su propia transformación, conducente a un compromiso ciudadano con la modificación de conductas, en un proceso pedagógico que facilita la creación de armas intelectuales y emotivas para una acción transformadora, consciente de la realidad.

Las anteriores perspectivas aportan elementos básicos a la educación ambiental preconizada, al favorecer que las personas tomen conciencia de los problemas ambientales concretos de su comunidad, a la vez que se estimula la creatividad. Estos aspectos permiten que se formulen propuestas de soluciones prácticas a estos problemas, sustentadas en su cultura y tradiciones, en un proceso pedagógico que supera la educación formal, para aprovechar todo momento o circunstancia del acontecer cotidiano; con la utilización de métodos que despierten la necesidad de ser autores y principales actores de su propia transformación.

Como se ha demostrado con anterioridad la educación ambiental debe extenderse a todos los sectores de la sociedad y por lo tanto expresarse a través de los ámbitos formales, no formales e informales. Se comprende por educación formal, la educación fuertemente institucionalizada, con objetivos y contenidos organizados en grados, niveles y especializaciones y cuyo accionar se extiende desde el nivel primario a la universidad, incluyendo la educación de adultos. La educación informal es aquella que no presenta un sistema de contenidos estructurados y se guía por objetivos generales, es propia de los medios masivos de comunicación.

El presente trabajo se inscribe en la educación no formal en un contexto rural, dado que está dirigida a un grupo social que en su mayoría no participa del sistema nacional de educación. Esta tiene el objetivo de formar un campesino con una cultura ecológica, con conciencia ambiental, sustentada en una racionalidad crítica 
y reflexiva, que sepa valorar y justipreciar la relación con su contexto.

Fernández (2008, p.34), define la educación no formal, como "un proceso educativo planificado, con carácter específico y diferenciado, generalmente dirigido a públicos heterogéneos propios de los procesos educativos comunitarios". Una definición más abarcadora la aporta Valdés citado por Bellido, (2007, p.65), al señalar que:

la educación no formal es un proceso pedagógico, que no exige una rígida institucionalización, aunque precisa de una organización que garantice la aplicación consecuente de las principales categorías didácticas, como objetivo, contenido, métodos y evaluación; con espacios de socialización y debates. Ella, generalmente está dirigida a grupos sociales heterogéneos con diversos niveles culturales y etarios, con el objetivo de prepararlos, para que sean capaces de enfrentar problemas que afectan la sociedad.

Al referirse al significado de este tipo de educación Delgado (2006, p. 69) expresa: "Los procesos educativos por las vías no formales cobran una especial relevancia, ya que estas modalidades tienen un impacto directo y una gran efectividad en todos los grupos sociales de la población."

En este trabajo se concuerda con los planteamientos anteriores, aunque se considera que debe explicitarse más el carácter pedagógico, para lo cual son necesarios espacios específicos de expresión del proceso de educación, formación y desarrollo, acorde con el contexto sociocultural donde se despliegue.

En los contextos rurales de la pesquisa, la educación no formal es asumida como un proceso dirigido a transformar conductas y desarrollar valores en grupos sociales heterogéneos, la mayoría de cuyos integrantes se encuentran fuera del sistema nacional de educación, como parte de un proceso educativo planificado, con carácter específico y diferenciado, con espacios determinados para la expresión del proceso de educación, formación y desarrollo, acorde al contexto sociocultural donde se extiende.

Las valoraciones realizadas permiten precisar la definición del concepto de educación ambiental, como un proceso pedagógico permanente e integral, basado en la educación no formal, capaz de generar conocimientos, habilidades y valores; modificar actitudes y conductas y permitir acciones conscientes y transformadoras, que conllevan a enfrentar y buscar soluciones a los problemas ambientales. Al tener la investigación como objeto el proceso de educación ambiental de la población campesina productora, se hace necesario valorar la problemática de la producción de alimentos en su relación con el medio ambiente.

En Cuba la producción de alimentos dirigida a satisfacer las necesidades de la población y sustituir importaciones es uno de los objetivos fundamentales. Lo anterior tiene como fin, sin negar la técnica, rescatar y desarrollar las tradiciones y las especies autóctonas. 
La agricultura moderna para incrementar la producción se fundamenta en el uso de productos químicos, empleo de maquinaria agrícola, formación de grandes empresas dedicadas a un solo cultivo (monocultivo) y la utilización de especies transgénicas. Este fenómeno ha sido conocido como revolución verde.

En sus inicios este tipo de agricultura logró un incremento de los rendimientos de los cultivos, pero con el transcurso del tiempo demostró su insostenibilidad al ocasionar pérdida de fertilidad de los suelos y de la diversidad biológica; altos niveles de plagas y enfermedades; deterioro medioambiental; incapacidad para asegurar la calidad de los alimentos y la capacidad del mercado; introducción de tecnologías incompatibles con los recursos de que se dispone y las características propias de cada región provocando un incremento en los costos de producción; serios problemas a la salud del hombre; pérdida o abandono de las prácticas o tradiciones culturales campesinas relacionadas con las producciones agrícolas.

Esta situación ha sido motivo de preocupación de científicos y ambientalistas, por lo que se preconiza un nuevo modelo de agricultura denominada agricultura sostenible, conceptualizada como aquella en la que los sistemas productivos permiten obtener beneficios continuos del agua, suelos, recursos genéticos, etc. para satisfacer las necesidades actuales de la población sin destruir los recursos naturales básicos para las generaciones futuras; al garantizar la seguridad alimentaria, la conservación de la naturaleza y el respeto al medio ambiente.

Los propósitos fundamentales de la agricultura sostenible son los siguientes:

- Garantizar la seguridad y autosuficiencia alimentaria.

- Conservar y regenerar los recursos naturales.

- Preservar la cultura local y de la pequeña propiedad.

- Alcanzar producciones estables y eficientes de los recursos productivos.

- Usar prácticas agroecológicas o tradicionales de manejo.

- Participación de la comunidad en todos los procesos de transformación agrícola.

- Aportar el bienestar a la comunidad.

Se trata de rescatar las prácticas tradicionales y la sabiduría campesina, conjugándola armónicamente con las técnicas modernas, devolviendo al campesino el papel principal en la producción agrícola y garantizar la sostenibilidad de los sistemas agropecuarios. 
Para lograr tal propósito en estos grupos de campesinos con carácter heterogéneos, los medios y métodos utilizados fueron propios de una educación no formal, que se sustentaron en conocimientos, habilidades y valores relacionados con el desarrollo agrícola y agropecuario, de sucesivas generaciones de campesinos, ofreciendo amplios espacios a la creatividad, sin negar la asesoría científica en la solución de los problemas ambientales, cuyo punto de partida lo constituyen las características propias de estos campesinos productores.

Para ello el personal empleado para emprender dicha tarea lo conformaron los activistas educativos. Fueron seleccionados entre los pobladores de las zonas rurales y suburbanas, entre los graduados universitarios, privilegiando agrónomos y maestros, dirigidos por estos investigadores. Otra forma la brindó la relación estrecha con la Facultad de Agronomía de la Universidad de Holguín entre los estudiantes de los últimos años de la carrera.

El número de activistas educativos se seleccionó a partir de la cantidad de cooperativas campesinas, donde cada activista atendió, cuando más, dos cooperativas, producto de lo numeroso de la población campesina y lo heterogéneo de su nivel cultural y actividades económicas. En la elección de los activistas se tuvo presente los siguientes indicadores: nivel cultural; conocimiento de la problemática del territorio y su impacto en el medio ambiente; conocimientos pedagógicos; capacidad de comunicación; voluntariedad y nivel de compromiso con la tarea encomendada.

La preparación comprendió los siguientes temas fundamentales: la problemática medioambiental y su incidencia en la agricultura en Cuba; situación y evaluación de la agresión al medio ambiente en la zona objeto de estudio; características y exigencia de la agricultura sostenible; fundamentos de la educación ambiental; principios en los cuales se cimienta la educación no formal y metodología a seguir para lograr con éxito la educación ambiental preconizada.

La preparación adoptó la forma de un curso de post-grado con conferencias y talleres. En este se debatieron los diversos contenidos y sus potencialidades de aplicación de la educación ambiental en el contexto estudiado. Como en el presente caso se inmiscuyeron los estudiantes de Agronomía de la Universidad de Holguín, se coordinó con la Facultad homónima, la Cátedra Paulo Freire y la Cátedra de Medio Ambiente, la instrumentación de un curso optativo y una asesoría que contó con el apoyo de profesores de esa Facultad.

El proceso que se inició con la selección de los activistas, continuo con el desarrollo del curso de preparación y se extendió a lo largo de todo el proceso investigativo, en el cual los activistas desarrollaron una ingente labor.

Las formas, para el desarrollo de la educación popular ambiental de carácter no formal, fueron variadas y se desarrollaron en coordinación con la dirección de dichas cooperativas, con el apoyo de las organizaciones políticas y de masas de la comunidad. 
Estas formas tomaron el carácter de: conversatorios, debates, intercambios de experiencias, proyección y análisis de videos e intercambio individual y familiar con los campesinos.

Los espacios se seleccionaron según las posibilidades y potencialidades del contexto y entre los mismos se pueden destacar: visitas a campesinos cooperativistas, reuniones ordinarias y extraordinarias de las cooperativas, acciones desarrolladas por las bibliotecas y otras instituciones culturales y educacionales.

Es preciso recordar que se está en presencia de una educación no formal, con contenidos y objetivos específicos, pero cuya aplicación ofrece gran flexibilidad. Por tanto la tarea más importante fue dialogar y convencer al grupo social de la importancia de la investigación, de la utilidad de la misma, tanto en el presente como para el futuro. Fue esencial que cada campesino se sintiera parte activa de la investigación, asumiera la significación del contenido conservacionista y estuviera consciente que su apoyo y conocimiento eran esenciales para resolver los problemas ambientales del contexto y asegurar una agricultura sostenible que afecte lo menos posible al medio ambiente y con ello elevar su calidad de vida.

Debe resaltarse que este proceso educativo exigió una nueva relación entre educador y educando, en la cual se revaloriza el conocimiento cotidiano y la experiencia del educando se toma como punto de partida para la elaboración de nuevos conocimientos, donde enseñando se aprende y el educando es valorado como un ser creador, capaz de generar importantes ideas, de transformación y auto transformación, en un proceso de democratización del conocimiento.

No puede olvidarse que el objetivo de este proceso educativo fue el empoderamiento del campesino, como resultado de la educación popular ambiental comunitaria, para que pudiera enfrentar la problemática ambiental, a partir de un cambio en el modo de pensar y actuar, sustentado en el desarrollo de actitudes de responsabilidad personal, de autonomía y de valores, como soporte de una transformación en la relación con el medio ambiente. Cambio favorecido porque se apoyó en la utilización consecuente de sus propios valores patrimoniales.

La evaluación partió de la valoración de las actividades realizadas por cada uno de los miembros del equipo y tuvo éxitos, dificultades y también iniciativa para superarlas. La evaluación de la marcha de la labor educativa entre los campesinos se realizó a nivel de cooperativa y tuvo forma de fórum comunitario. Con días de anticipación a la celebración del fórum se plantearon los temas del debate y se solicitó que se prepararan para su evaluación. Se hizo imprescindible recordarle a la población campesina que su conocimiento, producto de la práctica y la tradición, era fundamental para el éxito de la labor que se realiza, pues lo que se estaba produciendo era la construcción de un nuevo conocimiento. Que su conocimiento sistematizado en dualidad con el conocimiento académico se convertiría en una importante contribución a la Ciencia. 
Se le pidióa los campesinos que fundamentasen los éxitos obtenidos en la producción, que realizaran pequeños experimentos, a la vez se aplicara la autoevaluación, coevaluación y heteroevaluación para reconocer a los más destacados y a la vez determinar las principales situaciones problémicas.

Dado el carácter de evaluación cualitativa, a través de la participación y colaboración brindada, se pudo medir las transformaciones en los distintos individuos respecto a la protección y conservación del medio ambiente. Se les recordó que se estaba ante una evaluación permanente, con fuerte valor educativo que contribuía al desarrollo de actitudes y valores y que aún los aparentes estancamientos e incluso retrocesos podía ser el punto de partida del éxito.

La labor pedagógica de los activistas fue constatada y a través del programa de capacitación de los campesinos productores se les felicitaba por los éxitos que iban logrando y se trataron algunas deficiencias de contenido y de metodología, valoradas después de cada visita individualmente y en la reunión periódica con el grupo.

Algunos campesinos consideraron que poco le podrían aportar los jóvenes activistas. Fue necesario utilizar elementos de la Pedagogía Freiriana para convencerlos, como fue la educación dialógica. En la aceleración del proceso contribuyó, además, las vivencias de los activistas, ellos son nativos y viven en la zona, todos con relaciones familiares y de amistad entre los campesinos; hecho que favoreció la superación de barreras.

El tema más candente en los diálogos fue el de la contaminación del medio ambiente por productos químicos, particularmente la cuestión de la calidad y procedencia de los insecticidas. En este caso los campesinos plantearon que las instituciones del Estado no tienen el surtido y la cantidad de los productos necesarios para enfrentar, por ejemplo las cosechas, por lo que tienen que acudir a suministradores clandestinos, que además de precios exorbitantes, ofrecen productos de dudosa calidad y con posibilidades de ser altamente contaminantes, pero que la necesidad, ante el peligro de perder sus cosechas, los obliga a utilizarlos.

No se ha erradicado la utilización de productos químicos, pero ha disminuido la cuantía de su aplicación, utilizándose, cada vez más abonos orgánicos y controles biológicos. Los campesinos prestaron mayor atención a la calidad y origen de los productos químicos y la manera de manipularlos; paralelamente se recupera la utilización de alternativas no agresivas.

Entre estas alternativas los campesinos plantaron: la siembra de carreras de maíz intercaladas en sembradíos de ajíes o frijoles, para atraer las plagas; no plantar nuevos cultivos de hortalizas o leguminosas cerca de otros ya crecidos, pues las plagas que acompañan a la maduración de los últimos se trasmiten a los primeros; favorecer la concurrencia de las garzas blancas al proceso de preparación de la tierra y el desarrollo de los cultivos, por ser estas aves grandes consumidoras de insectos y 
roedores; separar los cultivos de los pastos por una zona arada libre de hierbas, para contribuir a obstaculizar el paso de los insectos.

Se han logrado avances en la protección de la tierra de laboreo, si bien, como tendencia se sigue rompiendo el potrero con tractor, se cuida que los arados de este solo remueven la tierra vegetal, las demás actividades como cruzado, surcado y aporques, preferentemente se realizan con bueyes, este último también se realiza con azadón. Se ha reactivado la tradición de no surcar acorde ni contra el declive del terreno, contribuyendo de esta manera a evitar la erosión, además se trata de evitar los arrastres de las corrientes de aguas situando frecuentes contenes en sus cursos.

Se recurre más frecuentemente a la rotación de los cultivos, en especial cuando se trata de ajo, cebolla o maní, sembrándose preferentemente en tierras en que antes se había cultivado fríjol, maíz o yuca. El barbecho se utiliza, cada vez más, para beneficiar tierras que han perdido parte de su capa vegetal y se le destina a la cría de animales, que con sus desechos orgánicos y los residuos de la alimentación paulatinamente recuperan el terreno.

Otro elemento que transformó el criterio de los campesinos, es el de la calidad de las simientes, tanto los tallos como las semillas, cuidándose de propagar las variedades más productivas, resistentes a las plagas y las altas temperatura, a la vez que se ha planteado la necesidad de recuperar variedades de especies de gran valor genético y subvaloradas por la producción comercial. Se ha rescatado la tradición del proceso natural de maduración de frutas, viandas y hortalizas para el consumo sin necesidad de emplear maduradores químicos que afectan las propiedades nutritivas de estos alimentos.

Sobre el tema de la afectación a la flora y la fauna los progresos fueron muy lentos, producto de la tradición campesina de la caza y captura de aves silvestres, la muerte de cualquier tipo de culebras que puedan detectar, en especial del majá de Santa María y la caza de la jutía Conga (relativamente abundante en la Sierra de Candelaria), también mueren muchas especies de animales como consecuencia de la contaminación de los alimentos y el agua después de haberse fumigado. Se considera que la actividad educativa debe ir, en este caso, acompañada por medidas correccionales, en la cual los guarda bosques deben tener un papel esencial.

Se considera que el mayor logro educativo que se ha alcanzado es que la comunidad campesina se está concienciando de la situación que viven y están desarrollando un tipo de explotación de la tierra sustentable en el tiempo; que debe existir una relación adecuada entre las actividades económicas, la tecnología que se emplea y la conservación del medio ambiente. En estos momentos el problema de la afectación al medio ambiente se ha convertido en un tema recurrente y de reflexión en las reuniones de los miembros de las cooperativas, donde se critican y se toman medidas contra las acciones que perjudican el entorno. 
Respecto al fórum realizado a los miembros de las distintas cooperativas de créditos y servicios, con el objetivo de conocer las opiniones de los campesinos sobre los resultados, los activistas recogieron las siguientes tendencias:

- Están satisfechos de las relaciones que se han establecido con los activistas, el respecto que muestran por los conocimientos de los campesinos y como no tratan de imponen ideas, sino que se esfuerzan por convencer.

- La mayoría se muestran preocupados por la incidencia negativa para su salud y la de los familiares que tiene el uso desmedido de los productos químicos.

- Afirman comprender que la agricultura intensiva es insostenible, que ya están experimentando sus consecuencias negativas, que hay que producir tratando de agredir lo menos posible el medio ambiente.

- Al descubrir el valor de su patrimonio agrícola, se sienten orgullosos del mismo, como herederos de una cultura milenaria.

- Muchos plantean que las directivas de las cooperativas deben de ser más exigentes con los que se preocupan solo de obtener dinero a toda costa.

- Agradecen los esfuerzos realizados para que ellos cambien su actitud inconsciente frente a la naturaleza .y reconocen que nunca antes nadie había tratado, con tanta claridad, con ellos el problema de la necesidad de la educación ambiental.

- En especial, los dirigentes plantean la necesidad que la Asociación Nacional de Agricultores Pequeños (Anap) y el Gobierno se involucren más en el enfrentamiento a los problemas ambientales de estos espacios sociales.

\section{Conclusiones}

En resumen múltiple han sido los autores que han conceptualizado el tema sobre medio ambiente y educación ambiental por lo que han mostrado sus preocupaciones y teorizado al respecto.

Este tipo de educación está dirigida a cambiar actitudes ante la naturaleza, pues se convierte en imprescindible en un mundo donde, producto del accionar no controlado de la sociedad humana ha puesto en riesgo al propio hombre.

La implementación de una adecuada educación ambiental en contextos rurales, donde viven campesinos pequeños propietarios es una tarea compleja, sus miembros presentan diferencias culturales, etarias y de intereses. Estos contextos 
están sometidos a un proceso de des-ruralización y la educación no formal es una vía para suplir estas diferencias y fisuras culturales.

La educación ambiental, contextualizada en San Andrés y Gibara, territorios holguineros, coadyuvó a la transformación de su campesinado, al concienciarse de la necesidad de proteger el medio ambiente a partir de una agricultura alimentaria sostenible y agroecológica.

\section{Referencias}

AGUILERA, Víctor Bellido. El árbol que silba y canta comprensión filosófica de la participación en el trabajo cultural comunitario, 2007. (120f) Tesis en opción al grado científico de Doctor en Ciencias Filosóficas -Programa modalidad libre, Instituto de Filosofía de la Universidad de La Habana, 2007.

ARRAGA, Leonel Palma de. Fortalecimiento de la capacidad interdisciplinaria en Educación Ambiental, España, n. 16, Revista Iberoamericana de Educación, 2005.

CASTILLO, Rafael Díaz. Hacia una didáctica del medio ambiente.1998. (70f). Tesis en opción al título académico de Master en Educación Superior. - Programa de Maestría de Educación Superior. Instituto Superior Pedagógico "Pepito Tey", 1999.

CASTILLO, Rafael Díaz. Hacia una didáctica del medio ambiente, Instituto Superior Pedagógico "Pepito Tey": Las Tunas: Editorial Universitaria, 1999.

CONSTITUCIÓN de la República de Cuba. Gaceta Oficial de la República, Editorial Política, La Habana, 2003.

DÍAZ, Carlos Delgado. Selección, compilación y Edición Científica, La Habana: Editora Félix Varela, 2006.

ESTRATEGIA Ambiental Nacional. La Habana : Editorial Academia, 1997.

ESTRATEGIA Ambiental Nacional 2007-2010. La Habana: Editorial Academia, 2007.

FERNÁNDEZ, Mercedes Iríbar. Modelo pedagógico para potenciar la educación ambiental de comunidades impactadas por el desarrollo de la industria turística. 2008. (120 f.) Tesis en opción al grado científico de doctor en Ciencias Pedagógicas. - 
Programa de doctorado modalidad libre. Universidad de Holguín, 2008.

Foro Internacional de Organizaciones no Gubernamentales y Movimientos Sociales. Tratados Alternativos de Río 92. Editorial Escafarlo, Bogotá, 1994.

IRÍBAR, Mercedes Fernández. Modelo pedagógico para potenciar la educación ambiental de comunidades impactadas por el desarrollo de la industria turística. 2008. (120f). Tesis en opción al grado científico de doctor en ciencias pedagógicas Programa de doctorado modalidad curricular, Universidad de Holguín, 2008.

LEY 33/ 81 de Protección del Medio Ambiente y del uso racional de los recursos naturales, La Habana: Gaceta Oficial de la República, 1981.

MCPHERSON, Margarita. La educación ambiental como vía de concreción de la interdisciplinariedad: Una aproximación desde la enseñanza. Aprendizaje de la ciencia, La Habana: Editorial Pueblo y educación, 2002.

NOVO, Miguel. La Educación Ambiental, bases éticas, conceptuales y metodológicas, Madrid: Editorial Universitas S.A, UNESCO, 1998.

UNESCO. Conferencia Intergubernamental sobre Educación Ambiental (1977, Tbilisi). Informe final, Paris, 1978.

VELÁZQUEZ, Víctor Bedoy. La historia de la educación ambiental: reflexiones pedagógicas, Universidad de Guadalajara: Editorial universitaria, 2004.

Recebido em julho de 2015

Aprovado em dezembro de 2015

Juan Carlos Rodríguez Cruz é doutor em Ciencias Pedagógicas e profesor da Universidad Oscar Lucero, Ciudad, Holguín (Cuba).E-mail: jcrodriguez@fh.uho.edu.cu

Luiz Bezerra Neto é doutor em Ciências da Educação e profesor da Universidade Federal de São Carlos (UFSCar), atuando na graduação e no Programa de Pós Graduação em Educação. É coordenador do Grupo de Estudos e Pesquisas sobre Educação no Campo E-mail: Ibezerra@ufscar.br 